\title{
Letter to the Editor regarding the journal article published in Abdominal Radiology "Reuse and reduce: abdominal CT, lumbar spine MRI, and a potential 1.2 to 3.4 billion dollars in cost savings"
}

\section{R. Kamanahalli ${ }^{1}$}

Published online: 1 January 2019

๑) Springer Science+Business Media, LLC, part of Springer Nature 2019

\section{Dear Editor,}

I read the fascinating article published in the Abdominal Radiology journal titled "Reuse and reduce: abdominal CT, lumbar spine MRI, and a potential 1.2-3.4 billion dollars in cost savings" [1]. I would like to congratulate the author of this article for a brilliant piece of research, which if translated into practice could have a tremendous impact in terms of huge cost savings to the health service.

However, I am concerned with the methodology used in this study that led to the results and the conclusion. The type of data in this study is clustered, i.e. multiple observations within each patient, that can be positively correlated. For example, foramen stenosis at L4-5 is often associated with similar findings at other levels in the lumbar spine. It is therefore necessary to perform either segment-level analysis or make appropriate statistical adjustments for multiple observations within the same patient to mitigate erroneous results. The authors have described 'per-patient' measures of sensitivity and specificity, for concordant and discordant cases, ignoring possible correlations between the observations. I would argue this method could potentially yield misleadingly high sensitivity and specificity in a small sample such as in this paper. The methods to calculate sensitivity and specificity of clustered data, adjusting for potential correlations between multiple observations within the same patient, have been previously described by Genders et al [2].

\section{References}

1. Klein MA. Reuse and reduce: abdominal CT, lumbar spine MRI, and a potential 1.2 to 3.4 billion dollars in cost savings. Abdom Radiol (New York). 2017 Dec;42(12):2940-5.

2. Genders TSS, Spronk S, Stijnen T, Steyerberg EW, Lesaffre E, Hunink MGM. Methods for Calculating Sensitivity and Specificity of Clustered Data: A Tutorial. Radiology. 2012 Dec 1;265(3):910-6.
R. Kamanahalli

Raghavendra.Kamanahalli@nhs.net

1 Northumbria Healthcare NHS Foundation Trust, North Tyneside General Hospital, Rake Ln, Tyne and Wear, Newcastle upon Tyne NE29 8NH, UK 